\title{
Proceso de consolidación; Retardo y Pseudoartrosis
}

\author{
Consolidation process; Delay and Pseudoarthrosis \\ Lizeth Hernández-Rosalio ${ }^{a}$
}

\begin{abstract}
:
The bone consolidation process is a topic of interest in the area of traumatology and orthopedics due to the constant incidence of fractures; so that the presence of various factors can lead to a delay in consolidation resulting in bone alteration. Being the delay in consolidation one of the main complications that occurs in fractures, so there are various theories of its etiology, with an inadequate vascularization of bone fragments at the fracture site, and the presence of excess movement in the affected region.

This study is based on updated scientific review sources where it is intended to make known how the consolidation process is carried out, therefore; the environmental, chemical, mechanical or genetic factors involved, as they could trigger a failure in bone healing; In this way, we can introduce ourselves to the study of the most common pathologies such as delayed consolidation and pseudoarthrosis. Make known the sites of injury with the highest prevalence, based on this help decision-making in order to provide a more accurate and effective approach and treatment to the patient; in this way to achieve an adequate function of the affected limb.
\end{abstract}

Keywords:

Fracture, Consolidation, Pseudoarthrosis, Treatment, Delay in Consolidation.

\section{Resumen:}

El proceso de consolidación de óseo es un tema de interés en el área de traumatología y ortopedia debido a la constante incidencia de fracturas; de modo que la presencia de diversos factores puede llegar a producir un retardo en la consolidación dando como resultado una alteración ósea. Siendo el retardo en la consolidación una de las principales complicaciones que se presenta en las fracturas así pues existen diversas teorías de su etiología, teniendo mayor aceptación una inadecuada vascularización de los fragmentos óseos en el sitio de fractura, y la presencia de exceso de movimiento en la región afectada.

El presente estudio se basa en fuentes de revisión de carácter científico actualizado donde se pretende dar a conocer cómo se lleva a cabo el proceso de consolidación, por consiguiente; los factores ambientales, químicos, mecánicos o genéticos que intervienen, ya que podrían desencadenar una falla en la consolidación ósea; de esta manera introducirnos al estudio de las patologías más comunes como son el retardo en la consolidación y pseudoartrosis. Dar a conocer los sitios de lesión con mayor prevalencia, con base en ello ayudar a la toma de decisiones para poder otorgar un abordaje y tratamiento más preciso y eficaz al paciente; de esta manera lograr una adecuada funcionalidad del miembro afectado.

\section{Palabras Clave:}

Fractura, Consolidación, Pseudoartrosis, Tratamiento, Retardo en la Consolidación.

\section{Introducción}

Los trastornos en el proceso de consolidación de las fracturas actualmente siguen representando un gran motivo de consulta en pacientes quienes sufrieron de fracturas, si bien no existe una etiología universalmente aceptada que produzca estos trastornos, existen diversas teorías postuladas acerca de la importancia de ciertos factores intervinientes tales como pueden ser mecánicos

\footnotetext{
a Autor de correspondencia. Universidad Autónoma del Estado de Hidalgo, https://orcid.org/0000-0001-7770-1735, Email: he375425@uaeh.edu.mx
} 
o bioquímicos y una vez presentes en la zona de fractura, se ha descrito su estrecha relación con un retardo en el tiempo previsto de restauración ósea o bien el fallo por completo del proceso de consolidación.

El proceso consolidación se lleva a cabo a nivel óseo en la zona de fractura y el principio más importante para que realizarse es una adecuada estabilidad de los fragmentos óseos; no obstante Giannoudis describió la presencia de cuatro factores pilares indispensables en el proceso de restauración ósea y los denomino como concepto "diamante" debido a que la alteración de alguno de estos conceptos nos daría como resultado un fallo en la consolidación ósea; dichos pilares son: a) células osteogénicas, que inician la reparación; b) construcción de un andamio oste conductor; c) fabricación de factores de crecimiento oste inductivos, como las BMP, y d) estabilidad mecánica. Para adentrarnos en los trastornos de la consolidación, debemos conocer cómo se produce dicho proceso de manera natural; es así como el presente estudio muestra las fases de la consolidación y acontecimientos que ocurren en ellas. ${ }^{(1)}$

\section{Consolidación Primaria}

La consolidación cortical, directa o primaria de una fractura, tiene lugar cuando se consigue la reducción anatómica de los fragmentos óseos; llevándose a cabo con poco o nulo tejido de granulación y ausencia completa de movimiento del foco de fractura, es decir aquella que sana de primera instancia sin la producción de callo óseo con apoyo de material de osteosíntesis. ${ }^{(2)}$

\section{Consolidación Secundaria}

La consolidación secundaria o indirecta es aquella que se produce en fracturas no estabilizadas quirúrgicamente; donde la mayoría de estirpes celulares tienen lugar y a su vez atraviesa cuatro fases con diferente tiempo de evolución y la presencia de diversos factores y mecanismo implicados tales como:

\section{Fase inflamatoria o Hemorrágica}

Consiste en el impacto y formación del hematoma; tiende a ocurrir en las primeras 24 a 48 hora y puede llegar a durar hasta de 5 días aproximadamente; se basa en un proceso hemorrágico e inflamatorio donde se inicia la activación y migración de células como macrófagos, leucocitos y linfocitos que acuden al sitio de lesión, formando un hematoma alrededor de la zona de fractura, una vez que el coágulo este consolidado puede llegar a desaparecer rellenando así la línea de fractura con fibrina (Ver Tabla 1). ${ }^{(3)}$

\section{Fase Reparadora o Callo Blando}

Se le denomina fase reparadora debido a que continúa la proliferación vascular y diferenciación de tejido conectivo de células osteogénicas madurando a condrocitos y osteocitos; el principal objetivo es la formación del callo, esta fase se subdivide en dos procesos denominados callo medular y callo perióstico cabe señalar que si existe la presencia de inestabilidad en el foco de fractura puede retrasarse entre 4 a 40 días aproximadamente. ${ }^{(4)}$

\section{-Callo Medular:}

Esta primera etapa del callo blando se produce en la capa interna o medular del hueso donde primero los fibroblastos depositaran células reticuladas y fibras colágena, luego; las células fibroblásticas acompañadas de brotes de vaso sanguíneos y capilares, avanzan hacia el foco de fractura. Dos semanas después, la medula es invadida por tejido vascular procedente de ambos fragmentos de la fractura dando como resultado la restauración de la circulación medular y los vasos nutricios.

\section{-Callo Perióstico:}

Se lleva a cabo desde el segundo día y comienza con la proliferación de las células osteógenas, localizadas en el estrato profundo del periostio. Una vez activadas continuara el proceso de reparación ósea y hacia los 10 días se ha formado ya un collarín de tejido muy celular alrededor de cada fragmento de la fractura. ${ }^{(5)}$

\section{Callo Duro}

En la formación de callo duro juega un papel muy importante la presencia de los osteoblastos y condroblastos debido a que se depositan en la masa hística en crecimiento para otorgar mayor estabilidad y soporte a los fragmentos de la fractura, ya que la presencia de movilidad no dejaría que se lleve a cabo esta fase de la consolidación. Es en este punto del proceso de consolidación donde podemos observar en imágenes radiográficas el progreso en la formación de callo perióstico, evaluando de tal manera la presencia de calcificación y osificación progresiva.

\section{Fase de Remodelación}

El proceso de remodelación se lleva a cabo en un periodo de tiempo comprendido entre 3 y 5 meses; consiste en la formación de un hueso inmaduro el cual se ve presente en huesos fetales y su presencia en el adulto es únicamente en el callo de fractura. Conforme el cartílago 
del callo es sustituido por hueso, este se va remodelando de manera gradual gracias a los osteoclastos, devolviendo al hueso su estado original por resorción de las trabéculas que ya no son necesarias. ${ }^{(6)}$

\begin{tabular}{|c|c|c|c|}
\hline $\begin{array}{l}\text { Fase de la } \\
\text { consolidación }\end{array}$ & Duración & Caracteristicas & $\begin{array}{l}\text { Hallazgos } \\
\text { radiologicos }\end{array}$ \\
\hline $\begin{array}{l}\text { Fase } \\
\text { inflamatoria }\end{array}$ & 5-6 Días & $\begin{array}{l}\text { migración de } \\
\text { estirpes } \\
\text { celulares, } \\
\text { angiogénesis y } \\
\text { edema. }\end{array}$ & $\begin{array}{l}\text { Difuminación } \\
\text { de Rebordes } \\
\text { Óseos }\end{array}$ \\
\hline $\begin{array}{l}\text { Fase } \\
\text { reparadora }\end{array}$ & 4-40 Días & $\begin{array}{ll}\text { Continua } & \text { a } \\
\text { proliferación } & \text { de } \\
\text { tejido de de } & \text { granulación. }\end{array}$ & $\begin{array}{l}\text { Formación } \\
\text { de callo y } \\
\text { calcificación } \\
\text { Ósea }\end{array}$ \\
\hline $\begin{array}{l}\text { Fase de } \\
\text { remodelación }\end{array}$ & $\begin{array}{l}3 \text { meses- } \\
\text { Años }\end{array}$ & $\begin{array}{l}\text { Síntesis y } \\
\text { remodelación } \\
\text { del tejido } \\
\text { osteoide. }\end{array}$ & $\begin{array}{l}\text { Trabéculas } \\
\text { Oseas en la } \\
\text { zona } \\
\text { medular. }\end{array}$ \\
\hline
\end{tabular}

Tabla 1. Fases de la consolidación ósea y sus principales características ${ }^{(5)}$

\section{Trastornos de la Consolidación}

Los trastornos de la consolidación son procesos muy frecuentes en el área de traumatología y ortopedia debido a su constante frecuencia, principalmente por el número de fracturas y la localización de ellas, siendo la tibia el hueso más comúnmente afectado asociado principalmente a una inadecuada inmovilización de la extremidad, permitiendo de tal manera la presencia de micro y macro movimiento en el foco de fractura. Si bien universalmente no existen definiciones específicas para describir estos trastornos; con base a su origen etimológico definiremos pseudoartrosis como "pseudo" que significa "falso" y "artrosis" "articulación", por lo tanto, podremos deducir que literalmente significa "articulación falsa" o bien es considerada como el fracaso definitivo de la osteogénesis en un periodo promedio mayor a 6 meses. ${ }^{(7)}$

Se habla de retraso en la consolidación de una fractura cuando pasa más tiempo de lo habitual para que el hueso se trate dando como resultado la presencia de un enlentecimiento en la velocidad de consolidación del hueso. Por otro lado, en la pseudoartrosis el proceso de consolidación ósea ha terminado, formando una cicatriz fibrosa; definitiva e irreversible es decir que la consolidación no se ha llevado a cabo correctamente y este proceso lo podremos visualizar por medio de imágenes radiográficas.

Los primeros datos de alarma que sugieren un retraso de consolidación ósea son bastante perceptibles siendo los más frecuentes: presencia de dolor en la zona de fractura, dificultad para realizar movimientos y la presencia de crepitación. ${ }^{(8)}$ Es importante recalcar que se trata de dos procesos diferentes debido a que sus signos y síntomas son similares y suele haber confusión a la hora de abordar el paciente. (Ver Tabla 2)

\begin{tabular}{lcc}
\hline Características & $\begin{array}{c}\text { Retardo de } \\
\text { consolidación }\end{array}$ & Pseudoartrosis \\
\hline Crepitación & +++ & + \\
\hline $\begin{array}{l}\text { Dolor } \\
\text { Movilidad del foco } \\
\text { de fractura }\end{array}$ & +++ & + \\
\hline $\begin{array}{l}\text { Engrosamiento de } \\
\text { los extremos } \\
\text { óseos }\end{array}$ & ++ & +++ \\
\hline $\begin{array}{l}\text { Esclerosis de } \\
\text { fragmentos }\end{array}$ & -- & + \\
\hline $\begin{array}{l}\text { Ausencia de } \\
\text { fibrosis marginal }\end{array}$ & + & -- \\
\hline $\begin{array}{l}\text { Obstrucción del } \\
\text { canal medular }\end{array}$ & -- & + \\
\hline
\end{tabular}

Tabla 2. Características Clínicas y Radiográficas en el Retardo de la Consolidación y Pseudoartrosis. (9)

\section{Incidencia}

Aproximadamente más de 2 millones de fracturas ocurren anualmente en México, de ellas el 6\% llega a desarrollar un retraso en su proceso de consolidación teniendo estrecha relación con el sitio de fractura, así como los factores de riesgo asociados que presenta el paciente. $(9,10)$

Se ha descrito mayor incidencia a desarrollar procesos patológicos de consolidación ósea en personas de raza blanca, con un rango de edad de 30-45 años, en extremidades inferiores; siendo más propensos los huesos largos con mayor afectación el tercio medial de su porción diafisaria teniendo una estrecha relación con los agujeros nutricios presentes en las diáfisis y el aporte sanguíneo que recibe. El hueso con mayor incidencia de fractura es la tibia por su localización subcutánea y pobre cobertura de músculos, de esta manera es considerada el hueso que más tarda en consolidar llevándole en promedio hasta 16 semanas. ${ }^{(11)}$

Se realizó un estudio retrospectivo-descriptivo publicado por revista cubana de ortopedia y traumatología ${ }^{(12)}$ donde a 80 personas que padecieron un proceso de pseudoartrosis en una zona de fractura el 66,25 \% de los pacientes lo presentaron en la tibia, posicionándose en segundo lugar el húmero con el 12,5\%, en tercer lugar, el 
fémur con un $7.5 \%$ y al final de la lista el cubito con tan solo el $7 \%$ de incidencia en orden de frecuencia.

\section{Etiología}

No existe etiología única que resulte específica, para que exista un fallo en alguna de estas etapas ya que deberán intervenir una serie de factores tanto externos como internos que altere el equilibrio en el proceso de consolidación.

Ramos, ${ }^{(13)}$ considera 3 grupos de causas:

1. Generales: Edad, Genero, Carencias Nutricionales, Tabaco.

2. Enfermedades Sistémicas: Diabetes, Lupus, Sífilis, Osteopatía Descalificante.

3. Locales: Traumatismos De Alta Energía, Fracturas Multisegmentarias, Fracturas infectadas principalmente gravedad de la lesión, Fracturas Abiertas, Disminución del aporte sanguíneo, Daño vascular y Nervioso.

4. Por tratamiento: Tratamiento de osteosíntesis inadecuado, una mala técnica de inmovilización.

Por otro lado, se ha descrito que la alteración de factores mecánicos y bioquímicos es necesaria para que exista un fallo; los primeros hacen referencia a la constante fuerza de tensión ejercida en la zona de fractura la cual impide una adecuada formación del callo ósea. Teniendo como base que los mecanismos de compresión favorecen la formación de callo óseo, por el contario a esto la constante movilización y las fuerzas de torsión impiden la adecuada formación de callo óseo.

Dentro de los factores bioquímicos descritos se encuentra la producción de colagenasa por los macrófagos y fibroblastos localizados en el foco de fractura. ${ }^{(14,15)}$

Todas estas en conjunto son alguna de las etiologías más relacionadas a la generación de un proceso inadecuado de la consolidación. (Ver Tabla 3)

\begin{tabular}{|c|c|}
\hline \multicolumn{2}{|c|}{ Factores de Crecimiento } \\
\hline Mitógenos & Factor de Crecimiento Fibroblástico \\
\hline Morfogenos & Proteína Morfogénica Ósea \\
\hline \multicolumn{2}{|c|}{ Factores Clínicos Generales } \\
\hline Edad & Adultos mayores \\
\hline Hormonas & Uso de corticoides y Esteroides. \\
\hline $\begin{array}{l}\text { Deficiencia } \\
\text { nutricional }\end{array}$ & Vitaminas $c, d, k$, calcio, proteínas. \\
\hline \multicolumn{2}{|c|}{ Fármacos Suministrados } \\
\hline Aines & Ibuprofeno, Diclofenaco \\
\hline Antibióticos & Ciprofloxacina \\
\hline Anticoagulantes & $\begin{array}{c}\text { Anticoagulantes orales y Heparinas } \\
\text { de bajo peso molecular }\end{array}$ \\
\hline \multicolumn{2}{|c|}{ Factores Externos } \\
\hline Tóxicos & Tabaquismo y Etilismo. \\
\hline Mecánicos & Fuerzas de cizallamiento y torsión \\
\hline
\end{tabular}

Tabla 3. Principales agentes que intervienen en el Proceso De Consolidación. ${ }^{(24)}$

\section{Sistema de Clasificación}

Las imágenes radiográficas del miembro afectado son el método más común, ampliamente usado, para diagnosticar y evaluar alguna patología en el área de traumatología y ortopedia no solo por su bajo costo a comparación de otros métodos de imagen sino por su alta sensibilidad y especificidad a la hora de evaluar una fractura. El canal medular es uno de los hallazgos que se evaluaran a la hora de buscar un proceso patológico de consolidación ósea apreciándose una zona radiolúcida en caso de que el canal medular se encuentre cerrado y el proceso de consolación haya concluido o abierto si solamente se tratara de un retardo en la consolidación; así como, como la presencia de descalcificación de los extremos óseos, ausencia de fibrosis marginal, o sombras de calcificación en partes blandas adyacentes a la fractura (callo óseo incipiente). ${ }^{(16)}$

A través del tiempo se han planteado diversas clasificaciones a partir de las imágenes radiológicas una de las principales es la clasificación de judet y judet, (17) que se basa en la viabilidad de los extremos de los fragmentos óseos y del aporte sanguíneo que se divide en:

\section{Por inestabilidad mecánica:}

Pseudoartrosis hipertrófica. Proceso mediante el cual el hueso intenta consolidar, pero por alguna falla en el tratamiento o por excesiva movilidad del foco de fractura no lo logra provocando una inadecuada vascularización de los extremos del hueso y en la radiografía podremos observar agrandamiento y ensanchamiento de los extremos óseos.

Esta a su vez se va a dividir en:

"pata de elefante" característica por su callo abundante e hipertrófico y "casco de caballo" que presenta una moderada hipertrofia a comparación de la anterior.

\section{Por falta de masa celular:}

Donde tiene lugar la pseudoartrosis atrófica o bien conocida como hipotrófica caracterizada fisiopatológicamente por una inadecuada irrigación del miembro; más comúnmente ubicada en el cuello femoral o cando existe una fractura abierta. En las radiografías observaremos adelgazamiento de los extremos óseos.

Schweiberer;, ${ }^{(18)}$ considera que las pseudoartrosis hipotróficas son pertenecientes al grupo de las biológicamente no reactivas descritas a continuación.

\section{Pseudoartrosis bilógicamente no reactivas; avitales 0 atróficas}

Se producen por la deficiente irrigación sanguínea que conllevará a una falla en la consolidación dando de esta manera falta de regeneración de estructura ósea, por lo tanto, no habrá callo. ${ }^{(19)}$

- Tipo a: con tercer fragmento: cuando un músculo no tiene inserciones, es distrófica. 
- Tipo b: necrótica (con mención de la zona).

- Tipo c: defecto óseo.

- Tipo d: atrófica (vía final común de toda pseudoartrosis).

La clasificación de paley y col. ${ }^{(20)}$ se basa en el defecto óseo existente:

- Con pérdida de hueso inferior a $1 \mathrm{~cm}$ :

- A1. Pseudoartrosis con deformidad móvil.

- A2. Con deformidad fija.

- Con pérdida de hueso superior a $1 \mathrm{~cm}$ :

- B1. Con defecto óseo.

- B2. Pérdida de longitud ósea.

- B3. Ambas.

\section{Diagnóstico}

Para realizar un adecuado diagnósticos de estas patologías es necesario recabar un conjunto de hallazgos principalmente de imágenes radiográficas y datos clínicos, sin embargo, no siempre podremos obtener una adecuada valoración por medio de las radiografías y en estos casos solicitaremos una Tomografía Axial Computarizada o una Resonancia Magnética que será de utilidad por su alto grado de sensibilidad a tejidos blandos, siendo de gran ayuda para detectar zonas de fibrosis y matriz cartilaginosa característicos de la fase regenerativa, o el líquido sinovial interpuesto que es sugerente de pseudoartrosis.

La ausencia de sombras de osificación adyacente al foco de fractura, separación de los extremos óseos, recalcificación, esclerosis, cierre del canal medular, así como engrosamiento de los extremos óseos por calcificación del tejido fibroso cicatricial son algunos de los principales hallazgos radiográficos que presentara un paciente con pseudoartrosis ${ }^{(21)}$

\section{Tratamiento}

Existen múltiples abordajes terapéuticos en los procesos patológicos de consolidación ósea, no obstante el principal manejo es realizar una adecuada inmovilización para evitar fuerzas de tensión que pudieran complicar el proceso, en la práctica médica resulta común que no se realice de manera adecuada dando como resultado el exceso de movimiento del foco de fractura; una vez asegurado la inmovilización de la zona de lesión se debe verificar si está presente un proceso infeccioso y tratar la causa de origen, así como corregir el estado nutricional del paciente.

El objetivo del tratamiento en pseudoartrosis es lograr una funcionalidad del miembro afectado lo más normal posible, mediante la resección de la cicatriz fibrosa.

Se realizó un estudio retrospectivo-descriptivo publicado por revista cubana de ortopedia y traumatología donde se evaluaron 11 casos de pseudoartrosis: 7 asépticas y 4 sépticas obteniendo como resultado que la fijación intramedular con clavos acerrojados es uno de los técnicas que mayor aceptación ha tenido en los últimos años, su elección depende de varios factores implícitos como la condición en la que se encuentre el tejido óseo y los tejidos blandos, tamaño del foco de fractura y posición de los fragmentos óseos y tipo de pseudoartrosis ya que se reportó que no se debe utilizarse este método en pseudoartrosis sépticas. ${ }^{(22)}$

Fue en 1895 cuando comenzó la aplicación de un nuevo método el cual se ha ido perfeccionando a través de los años y consiste en la fijación intramedular con aplicaciones de placas y tornillos, método que sigue siendo uno de los más aceptados hoy en día, su principal aplicación en huesos largos, con una restricción de no aplicar si existe una deformación severa de la extremidad; uno de los efectos negativos de este método resulta que a mayor grado de abordaje tiende a tener mayor índice de infecciones. ${ }^{(23)}$

Clancey y cols reportaron ${ }^{(24)}$ las principales dificultades que pueden llegar a presentar estos métodos; en su estudio describió que en la técnica de fijación intramedular aplicando el clavo de kunschert (tratamiento de elección en la mayoría de las fracturas diafisarias de los huesos largos), presenta la dificultad de tener que esperar hasta 2 meses más para que el paciente deambule. Por el contrario, con el empleo de la técnica de fijación externa se reportó mayor incidencia en defectos óseos, acortamientos o deformidades, sin embargo, la realización de esta técnica posee ventajas tales como una menor invasión del foco de fractura, y la posibilidad de deambular ligeramente al cabo de 3 o 4 días con ejercicios activos. ${ }^{(25)}$

\section{Discusión y Conclusiones}

Al producirse una fractura pueden existir complicaciones locales o extrínsecas relacionadas directamente con el proceso de consolidación de la fractura, en cualquier fase de su evolución. Es así como la reparación ósea puede tomar dos caminos: seguir un proceso reparativo exitoso o sufrir alteraciones causadas por una serie de factores dando como resultado un trastorno en el proceso normal reparativo.

Estableciendo claramente la diferencia entre retardo en la consolidación y pseudoartrosis; se logró identificar las principales manifestaciones clínicas y radiológicas en la presencia de un trastorno en la consolidación ósea; siendo las más frecuentes la presencia persistente de dolor, la dificultad en el movimiento y sus principales sitios de aparición como lo es la tibia. Su diagnóstico no es tan 
fácil de detectar al principio, porque su visibilidad a las radiografías depende de la fase y el grado de evolución en que se encuentren las líneas de fractura y la presencia de callo en el hueso.

Es importante señalar que el abordaje en cada paciente debe ser individualizado teniendo en cuenta todos los factores presentes para lograr lograr el objetivo terapéutico y la persona obtenga un mayor grado de funcionalidad del miembro afectado lo más normal posible para mejorar la calidad de vida del individuo.

\section{Referencias}

[1] Álvarez R, Ojeda M. Retardo de consolidación y pseudoartrosis. En Álvarez R, Ceballos A, Murgadas R. Tratado de cirugía ortopédica y traumatológica. La Habana: Pueblo y Educación, 1986; t 2:348-466

[2] Arias M, Cortez RMP, Novillo WXG, Pulua EER, Díaz-Granados DAR Torres AAM, et al. Uso de aloinjerto esponjoso en fracturas pseudoartrosis en defectos óseos: experiencia en el Hospital Luis Vernaza.2018. Redalyc.org. [citado el 12 de abril de 2021].

[3]. Auersperg V, Trieb K. Extracorporeal shock wave therapy: an update EFORT Open Rev. 2020;5(10):584-92.

[4] Azagra R, López-Expósito F, Martin-Sánchez JC, et al. Incidencia de la fractura de fémur en España (1997-2010). Med Clin (Barc). 2015;145(11):465-470. doi: 10.1016/j.medcli.2015.02.023. Epub 2019 May 13.

[5] Canavese F, Samba A, Rousset M. Pathological fractures in children Diagnosis and treatment options. Orthop Traumatol Surg Res. 2016;102(1 Suppl):S149-59.

[6] Carbó-Laso E, Sanz-Ruiz P, del Real-Romero JC, Ballesteros-Iglesias Y, Paz-Jiménez E, Arán-Ais F, et al. Nuevo método de liberación de antibióticos del cemento óseo (polimetilmetacrilato): redefiniendo los límites. Rev Esp Cir Ortop Traumatol. 2018;62(1):86-92.

[7] Delgado del Caño C, García López J, Rodríguez de Oya R Pseudoartrosis séptica. Actualización en manejo y tratamiento. Rev Esp Traumatol Labor. 2019; 2 (1). Disponible en: https://www.setla.es/wpcontent/uploads/2019/05/02103-pseudoartrosis-septica.pdf Arrebola C. Pseudoartrosis y retardo de consolidación. URL disponible en http://www.ortoinfo.com. (Fecha de acceso: diciembre del 2019).

[8] Eisenberg KA, Vuillermin CB. Management of congenital pseudoarthrosis of the tibia and fibula. Curr Rev Musculoskelet Med. 2019;12(3):356-68.

[9] Fortune J, Paulos J, Liendo C. Manual de Ortopedia y Traumatología Santiago de Chile: Facultad de Medicina, Pontificia Universidad Católica de Chile. URL disponible en http://escuela.med.puc.cl/paginas/publicaciones/textotraumatologia/ $\mathrm{Tr}$ au_Portada.html. (fecha de acceso: enero del 2007).

[10] Franco-de la Torre L, Villafán-Bernal JR, Garmendia-Castañón R, Franco-González AP, Isiordia-Espinoza MA, Alcalá-Zermeño JL, et al. La combinación de ruido y soporte de peso acelera el tiempo de consolidación en las fracturas de la diáfisis tibial: un informe preliminar. Cir Cir. 2019; 87 (1): 18-22.

[11] Garbayo Marturet A.J., Tejero Ibáñez A., Repáraz Padrós F.J., Castaño Serrano A.. Tratamiento de la pseudoartrosis recalcitrante de húmero en pacientes de edad avanzada. Anales Sis San Navarra [Internet].
2014 Ago [citado 2020 Jun 05] ; 37( 2 ): 257-264. Disponible en: http://scielo.isciii.es/scielo.php?script=sci_arttext\&pid=S113766272014000200009\&lng=es. http://dx.doi.org/10.4321/S113766272014000200009

[12] Gubin AV, Borzunov DY, Marchenkova LO, Malkova TA, Smirnova IL. Contribution of G.A. Ilizarov to bone reconstruction: historical achievements and state of the art. Strat Trauma Limb Reconstr. 2016;11(3):145-52.

[13] Gustilo RB, Mendoza Rm, Williams DN. Problems in the Management of Type III (Severe) open fracture: A new Classification of Type III open fractute. J Trauma. 1984; 4:724-6.

[14] Hak DJ, Fitzpatrick D, Bishop JA, Marsh JL, Tilp S, Schnettler R, et al. Delayed union and nonunions: epidemiology, clinical issues, and financial aspects. Injury. 2014;45 Suppl 2:S3-7.

[15] Jacobo Núñez ME, Álvarez Cambras R, Sánchez Noda EO, Marrero Riverón LO. Pseudoartrosis de los huesos largos tratadas con osteosíntesis e injerto óseo de banco de tejidos. Rev. Cubana Ortop Traunmatol 2004;18 (2).

[16] Lionel L, Barla JD, Diego C, Danilo T, Sancineto CF, Carabelli G. Treatment of infected pseudoarthrosis in a subtrochanteric fracture in a patient with osteopetrosis. Case Rep Orthop. 2020;2020:5630202.

[17] Marco-Martínez F. La fractura de cadera en el siglo XXI. ANALES RANM [Internet]. Real Academia Nacional de Medicina de España; An RANM . Año 2018 . número 135(03):203-210. DOI: http://dx.doi.org/10.32440/ar.2018.135.03.rev01

[18] Moon RJ, Harvey NC, Curtis EM, de Vries F, van Staa T, Cooper C. Ethnic and geographic variations in the epidemiology of childhood fractures in the United Kingdom. Bone. 2016;85:9-14. doi:10.1016/j.bone.2016.01.015

[19] Pancorbo Sandoval Enrique, Delgado Quiñones Alberto, Martín Tirado Juan, Hernández Hernández Justo, Díaz Prieto Giraldo, Quesada Pérez José. Tratamiento de la pseudoartrosis de los huesos largos mediante fijación externa. Rev Cubana Ortop Traumatol [Internet]. 2010 Dic [citado 2020 Jun 04]; 24( 2 ): 4456.

[20] Panja D, Kenney JW, D'Andrea L, Zalfa F, Vedeler A, Wibrand K, et al. Two-stage translational control of dentate gyrus LTP consolidation is mediated by sustained BDNF-TrkB signaling to MNK. Cell Rep. 2014;9(4):1430-45.

[21] Ruiz C, Pretell J. Pseudoartrosis diafisaria. Información personal no publicada

[22] Schaweiberer L, Baumgart R, Deiler S. The biological reaction in atrophic and hypertrophic pseudarthrosis of diaphysis of long bone. Causes and forms of appaearance. Chirurg 1999; 70(11):1193-201.

[23] Tagliapietra J, Fantoni I, Taglialavoro G, Angelini A, Berizzi A, Belluzzi E, et al. Symptomatic pseudoarthrosis secondary to a stress fracture of the acromion. Acta Biomed. 2019;90(4):603-5.

[24]. Taylor Ch. Retardo en la consolidación y pseudoartrosis. Campbell. Cirugía Ortopédica. Ed. Panam 8A. ed. P, 1212.

[25] Tiedeman J, Conolly J, Strates B, Lipiello. Treatment of non union by percutaneous injection of bone marrow and desmineralized bone matrix and experimental study in dogs. Clin Orthop 1991; (268): 294-302. 